\title{
When and Why Are Emotions Disturbed? Suggestions Based on Theory and Data From Emotion Research
}

\author{
Klaus R. Scherer \\ Swiss Center for Affective Sciences, University of Geneva, Switzerland \\ Department of Psychology, University of Munich, Germany
}

\begin{abstract}
Diagnosing emotion disturbances should be informed by current knowledge about normal emotion processes. I identify four major functions of emotion as well as sources for potential dysfunctions and suggest that emotions should only be diagnosed as pathological when they are clearly dysfunctional, which requires considering eliciting events, realistic person-specific appraisal patterns, and adaptive responses or action tendencies. Evidence from actuarial research on the reported length of naturally occurring emotion episodes (including potential determinants) illustrates appropriateness criteria for the clinical evaluation of emotion duration-an essential element in the DSM-5 symptom catalogue for major depression episodes, especially in bereavement. The need for more actuarial evidence on normal emotion responses and its consideration by the clinical community is highlighted.
\end{abstract}

\section{Keywords}

appraisal bias, dysfunctional emotional responses, emotion duration, emotion functions

Here is a provocative question: Would you let someone diagnose and repair a motor problem in your car if they had little knowledge about how different motors function normally? Chances are that you would be quite reluctant.

What about emotion disorders? Shouldn't we know how emotions function normally before we attempt to diagnose and repair them? It is a provocative question but central to the topic of this special section - the distinction between normal and abnormal or disturbed emotions. In this contribution I will argue that thorough knowledge and agreement about the nature and functions of emotion and empirical data on their manifestations are essential for any attempt to deal with this burning issue confronting psychiatry and clinical psychology. While this article has been motivated by the ongoing debate about the fifth revision of the DSM, no attempts will be made to cover the literature on the underlying issues given the extensive discussion of the debate in other articles in this special section. Rather, using a concrete model of normal emotion and some illustrative empirical results, an attempt will be made to develop some concrete criteria to diagnose inappropriate, disturbed, or maladaptive emotional responses.

In order to chart normal emotion processes, I will first give a brief overview of the emerging consensus in the affective sciences on the definition of emotion and the underlying mechanisms. This will be followed by some empirical data on the factors involved in the elicitation of emotion and its resulting quality, intensity, and duration including individual and cultural differences. I suggest these elements are useful when discussing issues around "normal" emotional responses and dysfunctional emotional responses. Emotions are not created equal. Given the complexity of these issues and the limited scope of this article, I will focus only on duration of emotional episodes to support my argument. This has particular relevance considering the central role of duration in the major ongoing debate around classification of normal grief and clinical depression. However, the intensity of emotional feelings, response patterns, and regulation efforts could be analyzed in a similar fashion.

Author note: This work was supported by funds granted to K. R. Scherer under Swiss National Science Foundation Grant (100014-122491) and European Research Council (ERC) Advanced grant PROPEREMO (No. 230331).

Corresponding author: Klaus R. Scherer, Swiss Centre for Affective Sciences, University of Geneva - CISA, Case Postale 60, CH-1211 Geneva 20, Switzerland.

Email: Klaus.Scherer@unige.ch 
There is increasing agreement on defining emotion as an interface between an organism and its environment, mediating constantly between changing events and the individual's responses. We can distinguish four major functions of emotion: (a) the evaluation or appraisal of events that happen to us in terms of their relevance and consequences for our needs, plans, and values, (b) the preparation, both physiological and psychological, of actions that are appropriate for dealing with or adapting to that situation, (c) the representation and regulation of our potential response to a situation where information acquired from steps (a) and (b) is integrated and represented in a central area of the brain that can then monitor and regulate emotion and (d) the communication of reactions, states, and intentions to other people (see Frijda \& Scherer, 2009).

One could argue that these four functions provide a solid basis to develop criteria to be used in the diagnosis of a disturbance of an individual's emotional responding, namely when someone (a) consistently evaluates the consequences of all kinds of situations in an incorrect or unrealistic fashion, (b) in consequence shows responses that are inappropriate to deal with the respective situations, (c) does not appropriately monitor and regulate the emotional reactions to change them accordingly, and (d) does not adequately communicate the resulting feeling state to the social environment. In all of these cases, separately or in combination, one would expect some degree of emotional dysfunction. As the disturbance factors linked to the major functions of emotion may have different origins and may respond to different remedial strategies, it seems essential to identify exactly what type of problem is involved.

Let us take a concrete example to illustrate how these functions provide potential criteria for understanding what is not normal or helpful in an emotional reaction. You walk along a hiking path and see a snake on the path. You stop immediately, your heart rate rises and your blood circulation and muscle tone change to prepare for flight, whilst a facial-vocal fright response warns your fellow hikers of the snake but also your emotional state. This would be a normal and useful, evolutionarily prepared, emotional response. If a second later, you realize that it is a plastic toy snake, you laugh and your physiological and psychological systems more or less revert rapidly to normal. Such a sudden fear response is one of the most rapid emotional responses and often one of the shortest as there are many cases of false alarm or effective coping responses. If, in such a case, the emotional response lasts too long even once you have realized the snake is a toy, this may be an indicator of an emotional disturbance and could be the sign of snake phobia. Another example is bereavement, following the death of a person close to us. This happens to all of us, and the appraisal of the ensuing consequences plays a major role for the nature of the emotional reaction and its duration. A prolonged period of grief is expected in all cultures. But the expectation is that the person will appraise this as a natural, uncontrollable event and turn the attention to the future. If, however, the person believes to be partly responsible or considers it impossible to adjust to the loss of the person, a severe long-lasting depressive mood might be considered as an abnormal or pathological response.
But how long is too long? As we shall see, it is difficult to give a normative answer to this central question. One important indicator might be the amount of psychological and physiological investment in relation to what is required to effectively deal with the situation. For example, stress usually occurs when one has to deal with an important task in a short period of time, requiring the mobilization of all mental and bodily resources. Such a high state of arousal is costly and, if it continues for a long time, may have serious health consequences. In the case of the snake, it may be justified to mobilize enough resources to allow us to run away but once we are safe or if we realize the snake is in fact a toy these resources should be freed up for other purposes. This therefore roughly defines the appropriate duration. Similarly, many other emotion episodes recruit resources and the longer they last, the more costly they become for the organism.

In normal functioning, this investment needs to be justified by the nature of the eliciting event and the difficulty and importance of dealing with it. I suggest that the diagnosis of an emotional disturbance is intimately related to the degree of justification of the emotional investment given the overall situation. Before this argument can be further explicated, it will be necessary to briefly review the emotion mechanism by presenting the component process model of emotion (CPM; see Scherer, 2001, 2009, for further detail).

\section{Description of the Component Process Model}

Figure 1 shows the architecture of the model, including the dynamic, recursive emotion processes following an event. Although the model is highly interactive I will describe it using the four main functions of emotion that I proposed in the introduction. In line with the dominant functional or adaptive approach pioneered by Darwin (1872/1998), emotions prepare readiness for action in terms of different, and possibly conflicting, action tendencies. The assumption is that the tendency to engage in highly emotional behaviors such as aggression or flight are prepared by emotions such as anger or fear but that their execution is multiply determined. Emotion is only one, albeit potentially important, factor, in addition to the situational context, strategic concerns, and the behavior of others.

As shown in the flow diagram, the CPM suggests that the event and its consequences are appraised on a set of criteria on multiple processing levels. The result of the appraisal will generally have a motivational effect, and this combined with the appraisal results will produce effects in the autonomic nervous system (e.g., in the form of cardiovascular and respiratory changes) and in the somatic nervous system (in the form of motor expression in face, voice, and body). All of this will be centrally represented in a multimodal integration area (with continuous updating as events and appraisals change). Parts of this central integrated representation may then become conscious and assigned to fuzzy emotion categories as well as being labeled with emotion terms. 


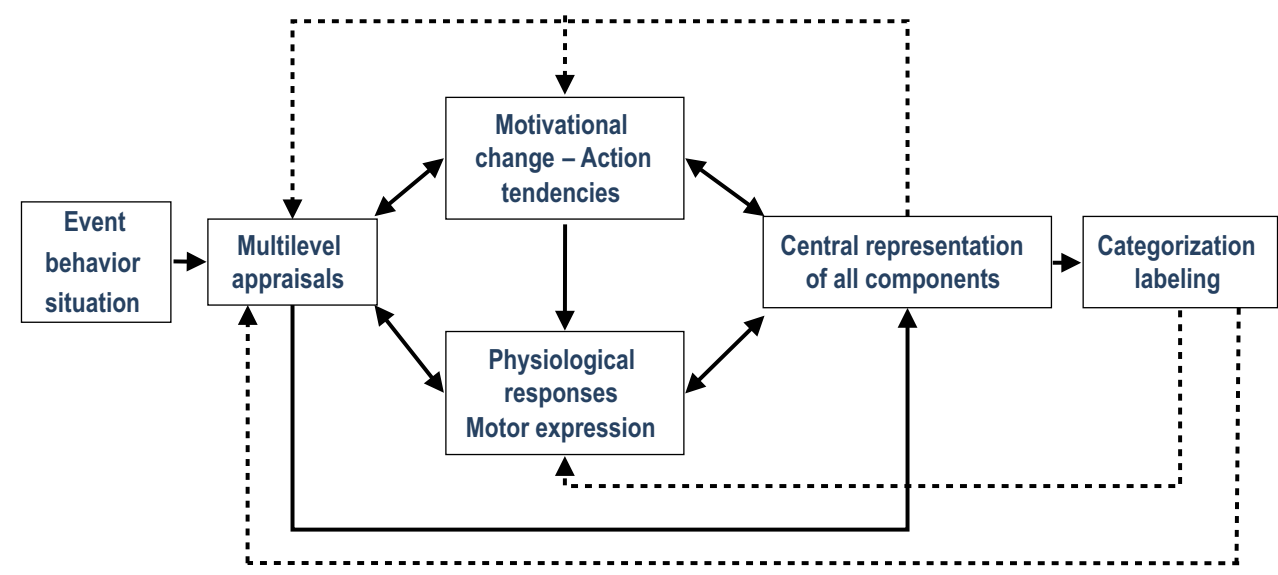

Figure 1. The dynamic architecture of the component process model (adapted from Scherer, 2009).

\section{Function 1: Evaluation and Appraisal}

The model suggests that there are four major appraisal objectives to adaptively react to an important event:

1. How relevant is this event for me and does it directly affect me or my social reference group? (relevance);

2. What are the implications or consequences of this event and how do they affect my well-being and my immediate or long-term goals? (implications);

3. How well can I cope with or adjust to these consequences? (coping potential);

4. What is the significance of this event for my selfconcept and for social norms and values? (normative significance).

Again these objectives can be demonstrated with the example of snake phobia where we appraise:

1. the likelihood it will bite in terms of proximity and possible type of snake (relevance);

2. the consequences of this bite in terms of whether it is poisonous (implications);

3. how well we can I cope with this in terms of possible options for fleeing (coping potential);

4. how significant it will be for self-concept and social norms in terms of whether and perhaps how others will view us if we run away (normative significance).

To attain these objectives, the person evaluates the event and its consequences on a number of criteria or stimulus evaluation checks (SECs). It is important to note that this is a subjective evaluation and may be more or less realistic. At any point of evaluation against the SECs, appraisal can be inappropriate or dysfunctional for example we can evaluate a small event to be overly important or we can minimize an important event. We can also assess it to have greater or lesser consequences than it does in reality or judge our coping ability as better as or worse than is actually the case. Lastly we can exaggerate or diminish the significance of an event for ourselves or our social group.

The appraisal process does not necessarily require a complex cognitive calculus. Processing can occur at different levels of processing such as (a) a low-level neural circuit in which the criteria consist of appropriate templates for pattern matching which may feed into disorders such as phobias (cf. the notion of "biological preparedness"; e.g., for snakes, Öhman \& Mineka, 2001; or baby faces, Brosch, Sander, \& Scherer, 2007); (b) a schematic level, based on memory traces from social learning processes which could be central to problems such as posttraumatic disorder; (c) a level involving various cortical association areas, which may occur automatically and unconsciously or in a deliberate, conscious fashion, which may play an important role in disorders such as anxiety and panic, and (d) the conceptual level, involving propositional knowledge, and underlying cultural meaning systems, requiring consciousness and effortful calculations in prefrontal cortical areas and could be important in problems such as antisocial personality disorder or psychopathy . The different levels continuously interact, producing topdown and bottom-up effects (see Leventhal \& Scherer, 1987; van Reekum \& Scherer, 1997).

\section{Function 2: Preparation for Action}

Reacting to events automatically with instinct-driven behavior only happens for a limited set of standard situations, such as withdrawing our hand when we burn ourselves. In specific circumstances this is useful but such a rigid mechanism would not allow flexibility for adaptation. The CPM proposes that emotions have evolved to decouple the trigger stimulus and the response, allowing the organism to rapidly prepare potential actions based on a series of SECs and to delay the actual response. As shown in Figure 1, the fundamental assumption of the CPM is that the appraisal triggers efferent outputs designed to produce adaptive reactions. Thus, emotion differentiation is the result of the net effect of all changes in cognition, motivation, autonomic arousal, and motor action brought about by the SEC sequence. 
The CPM makes specific predictions about the effects of the results of certain appraisal checks on the autonomic and somatic nervous systems, indicating which physiological changes and which motor expression features are expected to happen. The beginning of an emotion episode is defined by the synchronization or coherence of subsystems above a certain threshold and its end by the coherence dropping below threshold. The nature of the emotion episode is exclusively determined by the pattern of appraisal results and its variation over time driven by the recursively generated appraisal results. This produces many different emotion processes without clear categorical boundaries. However I also suggest the existence of certain modal outcomes that occur more frequently due to event contingencies and psychobiological prewiring, and which are verbally labeled in many languages (see Scherer, 2009).

\section{Function 3: Representation, Monitoring, and Regulation}

The CPM assigns a special status to the feeling component in the emotion process, which should not be confused with the complete componential emotion package (see Scherer, 2005a). The feeling component has the special function of monitoring and regulating the process and enables the individual to communicate an emotional experience to others. Subjective experience needs to integrate and centrally represent all information about the patterns of change. Figure 2 shows a proposal for a CPM account of how feelings integrate the central representation of appraisal-driven response in emotion (Scherer, 2004).

Psychologists generally assume that feelings are conscious phenomena, which can only be accessed and measured via verbal report. However, this widespread assumption holds only for the visible tip of a huge iceberg. The CPM (Scherer, 2004, 2005a) conceptualizes the problem, as shown in Figure 2, with a Venn diagram in which a set of overlapping circles represents the different aspects of feeling. The first circle (A) represents the sheer reflection or representation of changes in some form of monitoring structure in the central nervous system (CNS). The second circle (B), which partially overlaps with the first, represents that part of the integrated central representation that becomes conscious, corresponding to what is more generally called "feelings" or qualia. I have suggested that the degree of synchronization of the components generates this awareness (Scherer, 2005a). This proposed architecture is highly dependent on the processes of synchronization and integration within and between components which I define as the critical defining feature of emotion episodes. The rules that might underlie the integration at different points in the emotion episode are likely to rely on the relative weight given to the different components-appraisal, physiological responses, motor expression, motivation, and action tendencies. Research to examine the nature of this multimodal integration has hardly started. Empirical evidence could also provide understanding of when this integration and synchronization breaks down, either because the process itself does not function or because the relative weights are not appropriate or beneficial for the situation.

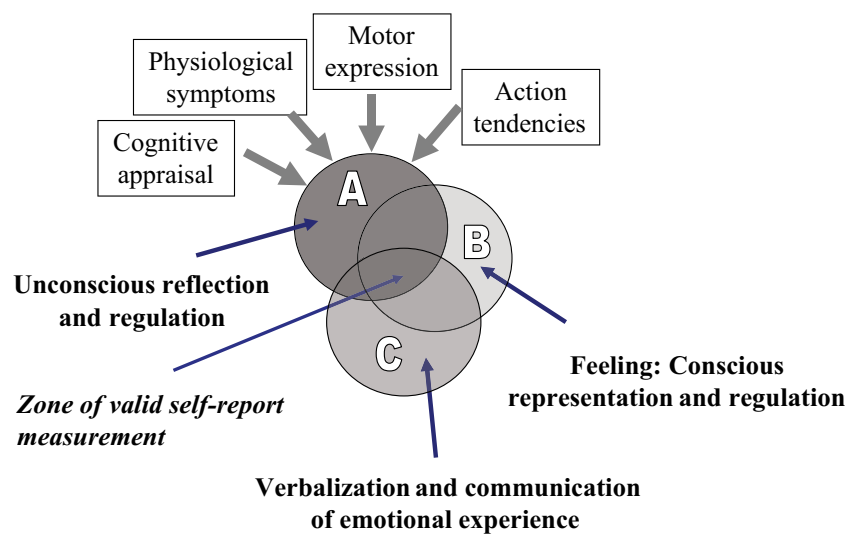

Figure 2. The reflection of component emotion processes in a monitor system (Circle A), the emergence of consciousness (Circle B), and categorization and verbalization (Circle C). (Adapted from Scherer, 2004.)

An important feature of emotion processes is their dynamic integration over time. Although humans can focus on micromomentary changes of feeling, we tend to become aware of our feelings in experiential chunks that provide phenomenal unity to a particular feeling quality or quale. It should be noted that this is an essential point for the issue of the duration of an episode - the reported length of an emotion episode is most likely determined by the subjective recall of the period of perceived or remembered synchronization.

Subjective feeling is the only place where all components of emotion are represented conjointly, allowing the type of ongoing monitoring which is essential for emotion regulation, and helping to settle issues like: Is my violent expressive reaction to a remark of my spouse in a dispute really justified by an appropriate appraisal of its meaning and consequences? Will this reaction deepen the conflict? Or would it be more promising to calm down?

\section{Function 4: Communication}

The communication of emotional experiences for adaptive purposes linked to group interests-informing others about our reaction to ongoing events and our behavioral intentions - often occurs through nonverbal channels via bodily expression, as in the case of most mammals. However, frequently humans also use language, particularly with the aim of the social sharing of emotion (Rimé, 2009). It is an interesting and complex issue to examine how preverbal feelings are labeled for communication. Presumably, the same experiential chunks that form the coherence clusters serve as the basis for verbalization. Again, the partial overlap of Circle $\mathrm{C}$ in Figure 3 indicates that the use of linguistic labels or expressions to describe the conscious part of feeling rarely covers the complete conscious experience. Verbal report always relies on language and the available words and expressions constrain the potential complexity of differentiation, which may account for much of the variance in emotion reports. An interesting question is whether the act of categorization may impoverish the rich qualia experience and mold it into 


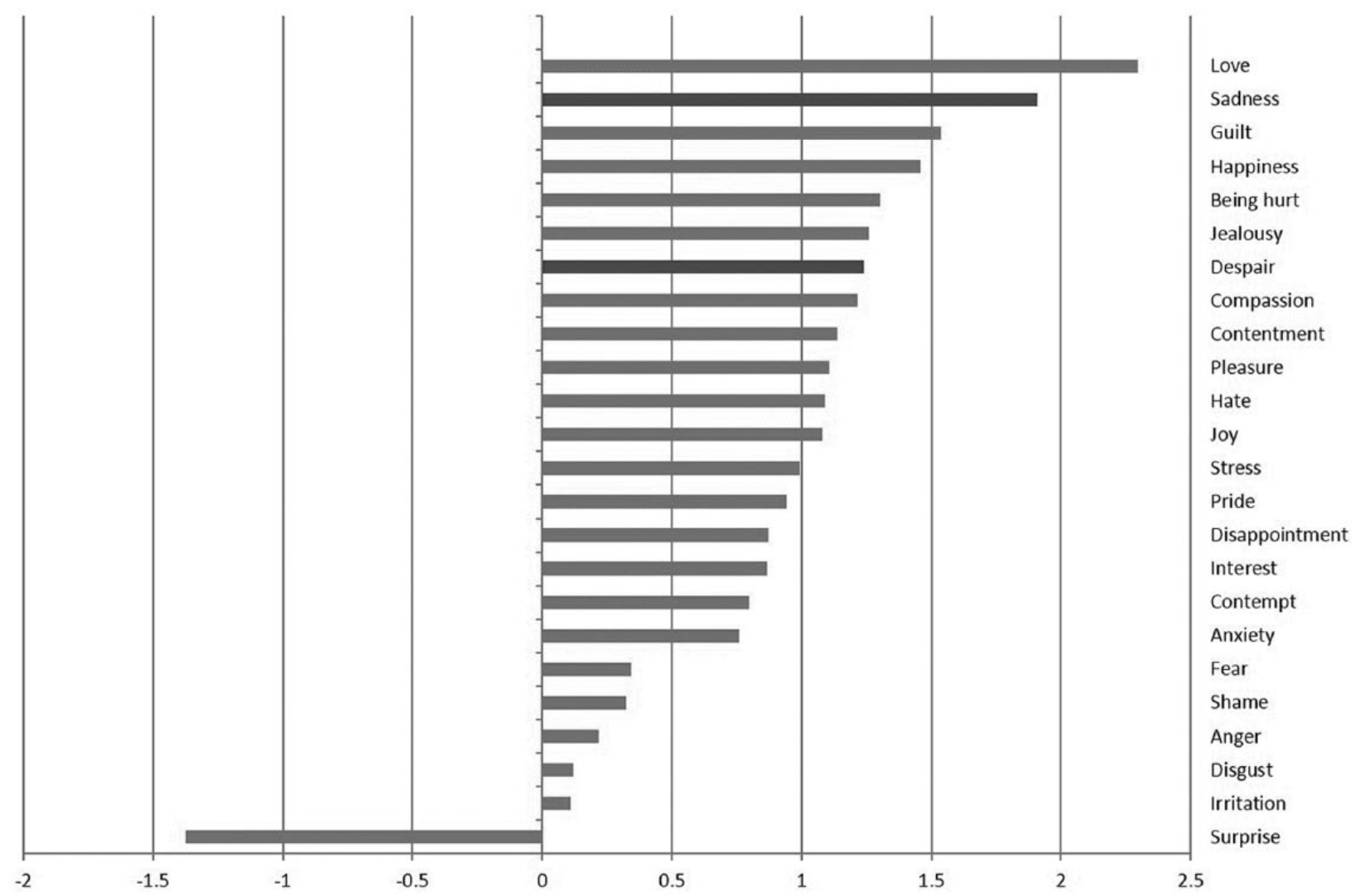

Figure 3. Differences in emotion duration as measured via semantic profiles of major emotion terms across 27 countries and 24 languages (standardized scores-computed separately for each emotion; for details and additional data analyses, see Fontaine, Scherer, \& Soriano, 2013).

socioculturally determined schemata. This is true for selflabeling and the recollection of memories based on labels as well as for other labeling. Thus, the issue of powerful, and often nefarious, effects of receiving diagnostic labels of affective disturbance (see Goffman, 1963) is of obvious importance for the issues under debate.

In both research and clinical practice, we rely on self-report and thus, the precise use of the emotion words is essential, especially given the rather small degree of overlap between unconscious representation, conscious feeling, and semantic meaning (the three circles in Figure 3). The degree of overlap may vary greatly between people and situations as can the accuracy in the communications of their emotional experience. The changes in the different components during the emotion episode only partly determine the conscious feeling and the label reported by the individual, particularly with respect to quality, that is, what kind of emotion-for example, melancholy, sadness, depression, shame - as well as its intensity and duration, depend on many other factors, such as verbal competence (for example, an individual suffering from alexithymia will have serious trouble describing his/her feelings in an accurate, fine-grained fashion).

\section{Duration of Emotion}

In what follows, I will use the example of the duration of emotion episodes to illustrate the value of placing emotional dysfunction within a model of normal emotion functioning. The aim is to illustrate the importance of understanding how long normal emotions last (especially the differences between different types of emotions and the potential causal factors determining duration) and why it might constitute a disturbance if their duration is too long or too short to be adaptive. This is one of the criteria for abnormality used by clinicians and lay people alike, as there seems to be an implicit assumption that a certain duration is appropriate for certain emotions. It is particularly salient in the context of the abolishment of the bereavement exception clause in DSM-5, given the general DSM philosophy to give great weight to the duration of symptoms and considering a 2-week period as sufficient to diagnose affective disorders (see the contribution by Horwitz, 2015). I will explore this issue by reviewing evidence from cross-cultural and cross-linguistic research on actuarial recall of emotion experiences and on semantic profiles of major emotion terms. The aim is to better understand the time frames in which different emotions normally arise and subside.

One can argue that the words in a language represent a "sedimentation" of human experience across the millennia and thus provides important insights into the phenomena they refer to. We have developed a GRID approach which asks native speakers to evaluate emotion terms on a feature vector of 144 items representing all components of emotion (Scherer, 2005b). Currently, data for 24 languages have been collected in 27 
countries (Fontaine et al., 2013). The results show that, while there are interesting cross-cultural and cross-linguistic differences, the meaning of emotion words is quite similar around the world. In particular, four dimensions define the affective space onto which we project the major individual emotion termsvalence, power/control, arousal, and unpredictability. Of course it is difficult to say what is a correct or incorrect projection of emotion onto these four dimensions but it does indicate that we can miscommunicate our emotions in terms of any of these dimensions, for example we can describe it as too strong or too out of our control and we can also suggest that it was completely unpredictable or that we are more aroused than is actually the case.

This research provides strong evidence that the semantic profiles of emotion words contain information on the culturally expected features on all emotion components for different emotions (appraisal, bodily reactions, motor expressions, action tendencies, and feelings). Important aspects of the feeling component are the intensity and the duration of the subjective feeling. Figure 3 shows the relative mean duration of different emotions found in the large-scale GRID study (for details see previous lines and Fontaine et al., 2013). Apart from love (reflecting the fact that the word suggests a long-term affective relationship rather than an individual emotion episode), sadness is by far the longest emotion closely followed by despair. As expected, we find several significant cross-cultural differences in rated duration but sadness is universally considered to be much longer than other emotions (with the exception of love). This suggests that a central tendency for what is considered a normal or appropriate length for different emotions is already encoded in our emotion vocabulary.

Self-report data from so-called actuarial research, in which people are asked to recall and describe emotion episodes they experienced in the recent past, can provide more precise information about the actual length of felt emotions. For the purposes of this article, I reanalyzed the data from a number of our earlier studies in which subjective duration reports were given on forced-choice scales.

Scherer and his collaborators (Scherer, Wallbott, \& Summerfield, 1986; Emotion and Social Interaction Study [ESI]) asked about 800 students in eight European countries to remember recent episodes of joy, anger, fear, and sadness and found significant emotion differences in terms of duration (fear $<$ anger $<$ joy $<$ sadness). In a large majority of cases sadness lasted for several days or longer while other emotions rarely lasted more than a few hours (1986, pp. 132-133). In a subsequent crosscultural study (the International Survey on Emotion Antecedents and Reactions, ISEAR; Scherer \& Wallbott, 1994) Scherer and his collaborators asked nearly 3,000 students in 37 countries to remember recent episodes. As shown in Figure 4 (ISEAR data), sadness was again the longest emotional experience, with more than $70 \%$ of the respondents reporting that it lasted weeks rather than days (more information about the ESI and ISEAR studies and a more detailed figure of the ISEAR duration results can be found in Section A of the Supplementary Material).

It could be argued that these results are not representative for the population as a whole as participants were students who might not represent all social classes. In addition, they are relatively young which might limit the range of their emotional experiences. Critics could also object that asking for specific types of emotion might have biased the recall as participants may not have an accurate memory of emotion episodes by category. To remedy these limitations, we conducted a semirepresentative anonymous survey, asking randomly selected Swiss citizens $(N=1,242)$, both from the German and the French part of Switzerland, to recall the most important emotion they had experienced yesterday and describe the event, their appraisal of the consequences, and their response patterns, including a verbal label. The results on the reported durations show that both sadness and despair had by far the highest intensity and the longest duration of 38 spontaneously mentioned emotion types (together with happiness/joy). It is interesting to note that this pattern was much stronger for respondents that were older than 40 years (more information about the study and a detailed data table can be found in Section B of the Supplementary Material).

A limitation of this representative study of everyday emotion experiences for the duration issue is that reporting the most important emotion experienced yesterday does not yield durations longer than several hours. A general limitation in the studies reported so far is that we did not provide a sufficient number of answer alternatives for duration allowing to capture emotions perceived to be lasting for rather long time periods. This was remedied in a large-scale web-based study using the Geneva Emotion Analyst (GEA). This expert system program (http:// www.affective-sciences.org/webexperimentation) proposes to automatically predict the best label for a given emotion experience on the basis of recalled appraisals, with surprisingly good results: only $10 \%$ of the participants consider the diagnosis as completely wrong (Scherer \& Meuleman, 2013). The program requires participants to enter a brief description of an emotion situation and answer a set of question on their appraisals and feelings including the duration of the respective emotion in terms of seconds, minutes, hours, days, and weeks. Figure 4 shows the results (GEA data) and in line with earlier studies we find sadness and despair to be by far the longest emotion experiences, with almost half of the respondents reporting a duration of several weeks (more information about the study and more detailed figures can be found in Section C of the Supplementary Material).

In general, these results show a very consistent pattern of emotion differences in terms of duration and show that in the sadness family, including grief and despair, a duration of several weeks may be the norm rather than an exception. Data from several actuarial studies on self-reports of spontaneously experienced emotions converge on the finding that normal duration of emotion episodes varies widely across individuals and emotions and it is not exceptional for certain types of emotional responses, particularly the members of the grief-sadness family, to frequently last over several weeks.

The results reported before have been obtained with a fairly representative sample of the population as different cultures have been involved and in two of the studies large samples of different age and occupational groups have participated. It can 


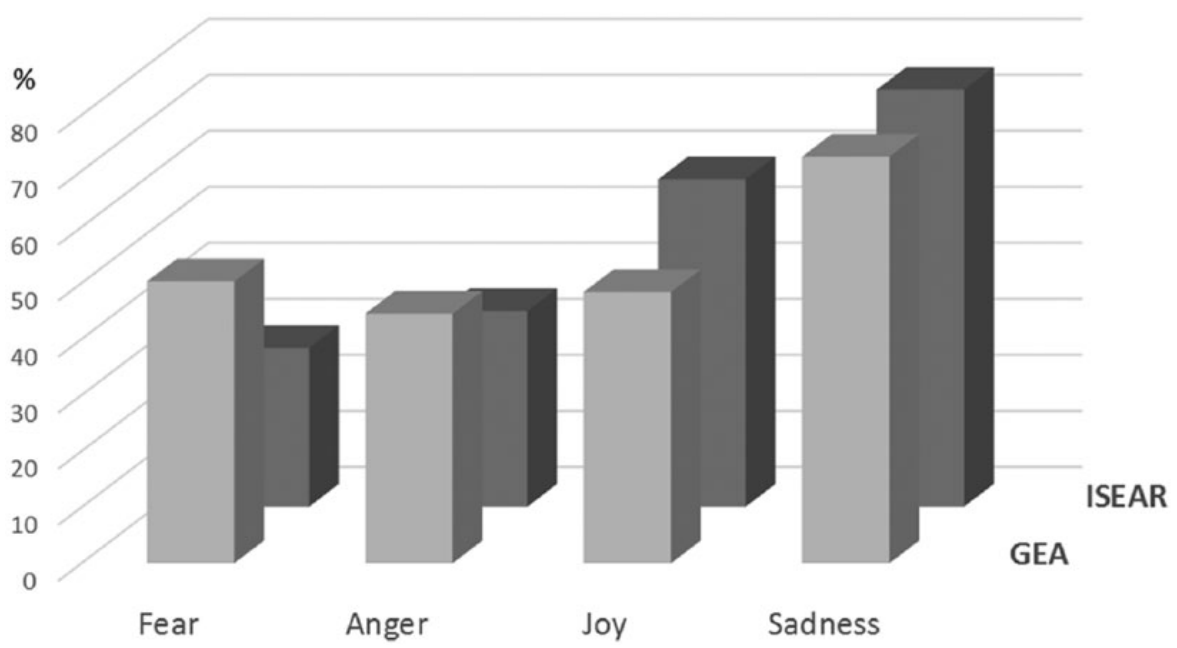

Figure 4. Differential duration of felt emotion in two cross-cultural self-report studies in percent of the respondents reporting that it lasted weeks rather days (ISEAR and Geneva Emotion Analyst [GEA] samples).

be argued that no clinical samples have been included and that it needs to be empirically shown that the results can be generalized. However, the purpose of the preceding review was not to suggest normative emotion durations for different groups. Rather the points to be demonstrated were that (a) even for normal populations there are important individual differences in duration for sadness and grief episodes-suggesting that it is difficult to impose a normative duration for a bereavement episode and (b) that sadness and despair last longer than most other emotions and that it seems not uncommon for bereavement episodes to last several weeks - suggesting that the standard 2-week period allowed by DSM-5 to the bereaved to return to normal before risking to be diagnosed with a major depressive episode may be unrealistic. In fact, the authors of DSM-5 acknowledged this and added a footnote encouraging psychiatrists to exercise clinical judgment in making the critical distinction between the symptoms characteristic of bereavement and those of a major depressive episode (see also Bondolfi, Mazzola, \& Arciero, 2015).

I suggest that this clinical judgment could greatly benefit from the results generated by research on normal emotions, especially research on grief and bereavement. For example, in an extensive review of relevant research, Stroebe, Schut, and Stroebe (2007) report that the first 6 months after the death of a close person are critical for the risk of increased physical pain, psycho-social symptoms, and mortality. Little seems to be known about the first few weeks after the onset of bereavement which is of central relevance for the diagnostic criteria of the DSM-5. We have started to use survey data to obtain some empirical evidence on this issue. Many countries have regular "household surveys" in which members of a large, representative panel of households are regularly interviewed about a wide range of topics concerning living conditions. They usually include detailed income information, financial situation in a wider sense, working life, housing situation, social relations, health, and biographical information of the interviewed. We have examined the data in the Swiss Household Panel (SHP $\left.{ }^{1}\right)$ which includes a number of interesting psychosocial variables, including emotional responses (http://forscenter.ch/wp-content/ uploads/2013/11/SHP_USER_GUIDE_W14.pdf).

Using data from the annual interview waves from 2006 to 2012, we examined the answers to a group of questions on the occurrence of a death by a closely related person, and if this was the case when exactly the death occurred, and to what extent the interviewee still suffered from this death (using an 11-point scale from 0 meaning "not at all" and 10 "a great deal"). We then treated each death as a separate event (independent of the year and whether the same interviewee had encountered several deaths in the course of the 7 years), computed the distance in weeks between the date of the death and the date of the respective interview, and plotted the relationship of the degree of suffering (defined by the percentage of interviewees reporting values of 7 or more on the intensity scale) to the weeks elapsed since the event. Visual inspection of the results for $N=6,214$ events, shown in Figure 5, suggests that there is a strong peak during the first week after the death, followed by an elevated level during a period of about 4 weeks, after which the average level of residual suffering does not descend much further. We used quartiles of the frequency distribution over week to form the following five temporal distance groups (subdividing the first quartile into two subgroups based on the pattern in Figure 5-less than 6 weeks, 6-10, 10-24, 24-39, more than 39) and computed the difference for the raw scores in residual suffering with a univariate ANOVA. The differences in suffering due to temporal distance were highly significant $(p<.0001$; eta square .026). A post hoc analysis using Tukey's HSD criterion showed three homogeneous groups (less than 6 weeks $=5.55,6-10$ weeks $=4.71$, and more than 10 weeks $=4.01,4.05$, and 4.34). The detailed data are shown in Supplemental Material D. It is interesting to observe the empirical distinction of different phases in bereavement suffering, suggesting a high level of suffering in the first 5 weeks and another 5-week period before reaching a stable baseline, which seems 
60

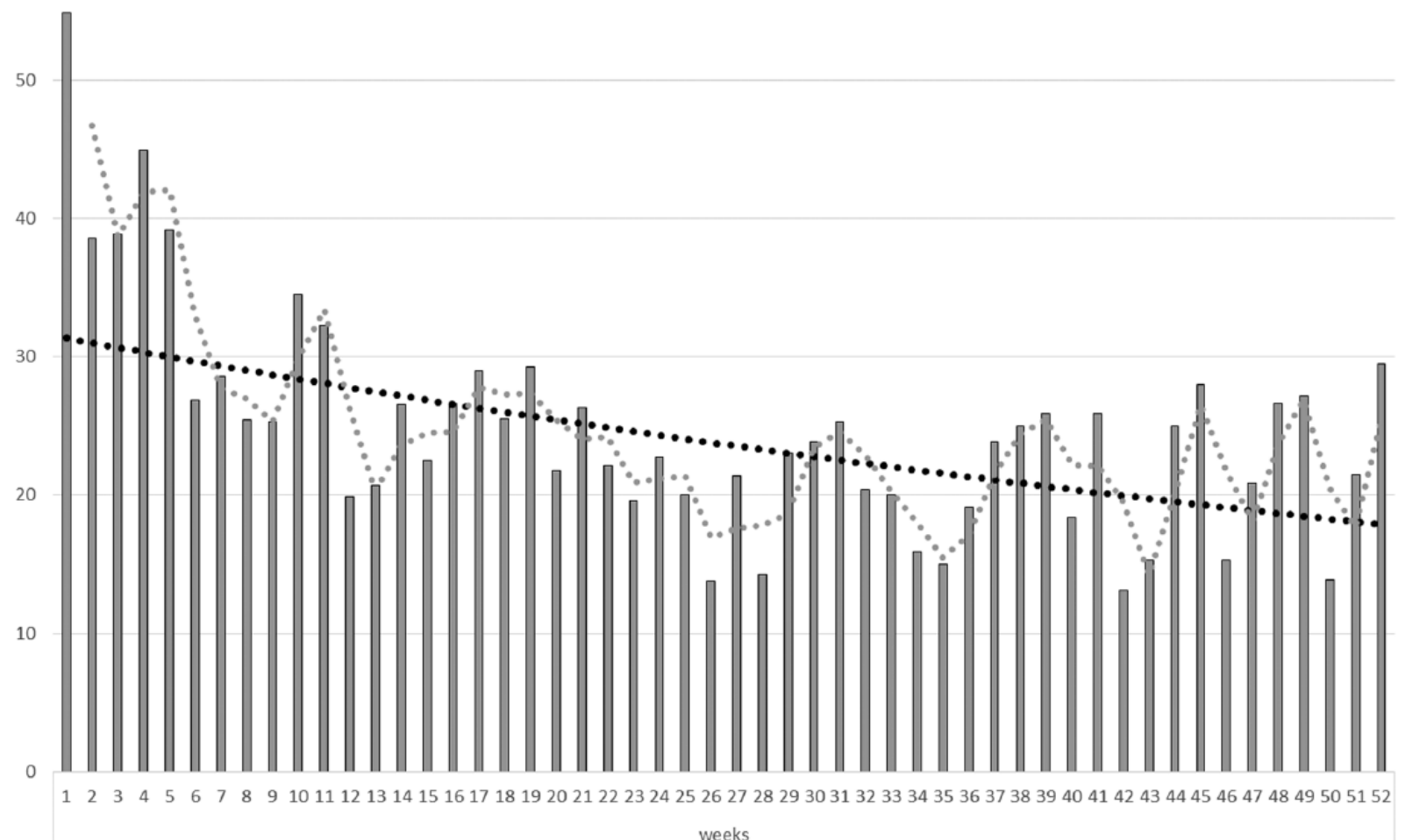

Figure 5. Percentage of bereaved respondents in the Swiss Household Panel suffering strongly (> 7 in an 11-point scale) as a function of weeks passed after the date of death of a close person (showing both a curve for the moving average-small dots-and the exponential trend line, bold dots).

quite at odds with the 2-week period suggested by the DSM-5. However, as one might expect, and as shown by the low effect size, there are important individual differences accounting for the strong variation in the data and we are currently developing structural equation models to analyze the relative effect of variables such as demographics, personality, values, and self-perception on the emotional response to bereavement. Preliminary latent class analyses suggest that a central factor might be a disposition to experience negative emotions.

The review of empirical data on emotion duration should not be seen as an attempt to establish a universal chronology of emotion processes. It just illustrates that emotions vary greatly in length and that emotions in response to loss such as sadness and grief generally take much longer to subside. The most important insight from the data sets discussed is the extreme degree of individual differences in emotion duration and intensity. As argued before, in order to understand the factors underlying the severity and duration of emotional responding and to diagnose dysfunctions, we would need a more profound understanding of the normal emotion mechanisms and the duration, intensity, and quality of the resulting reaction patterns. The emotion model proposed at the outset, the component process model, predicts that it is the differential appraisal of eliciting events, their consequences, and the coping potential of the person that determine the nature, intensity, and duration of the emotional episode, and this has been largely corroborated by empirical evidence (see Ellsworth \& Scherer, 2003; Scherer, 2009 , for an overview). Using such an approach could also help to find principled ways to understand and possibly justify longer durations of emotional symptoms for particular individuals.

In consequence, my claim is that the diagnosis of certain emotional response patterns as dysfunctional should be based on the appraisal processes that initially produced the symptoms. Thus the issue of normality versus abnormality shifts from exclusive concern with symptoms to include an analysis of the causes in terms of the appropriateness of the appraisal process. Before discussing and justifying this claim further, I would like to briefly review the empirical evidence for the central role of appraisal in the duration of emotions. In several of the studies reported before we were able to run regression analyses to determine whether reported length of experience could be predicted from the responses concerning the type of event or the nature of appraisals.

In our first actuarial study in eight European countries (ESI; Scherer et al., 1986; see preceding section) we asked respondents what type of event caused the emotion episode. Table E1 (in Supplemental Material section E) shows the frequencies with which different types of events or situations elicited joy, sadness, anger, and fear. The table shows that the major elicitors of emotions from the sadness family are deaths, relationships, and separation (Wallbott \& Scherer, 1986). These are clearly the types of events that will be appraised as the most relevant, 
producing serious consequences with little coping potential. It seems likely that high intensity and prolonged duration of these episodes are due to the perceived importance of these elicitors for a person's life and well-being.

This interpretation is supported by a result of a stepwise regression analysis for the data in the ISEAR study (a new, additional data analysis, shown in Figure E1 in the Supplemental Material) that shows the relative importance of the results for different appraisal checks (indexed by the size of standardized beta coefficients and size of sequential contribution to the varianceR2 coefficients) on the duration of reported sadness durations. The results show that more unpleasant, more immoral, and goalobstructive events combined with lower coping potential of the person typically result in longer duration of emotions. A separate reanalysis of the ISEAR data set using multilevel analysis revealed that negative emotions last especially long the more the eliciting event and its consequences are perceived to be incongruent with the individual's goals, values, and self-ideal, creating a mismatch. This relationship was largely stable across all cultures studied (Verduyn, van Mechelen, Tuerlinckx, \& Scherer, 2013).

A similar analysis was performed for the data collected with the GEA expert system, using slightly different appraisal questions (a new, additional data analysis, shown in Figure E2 in the Supplemental Material). Here, the results show that high goal relevance and unpleasantness of the event as well as low coping potential (in this case expressed by the fact that other people or fate rather than the person are seen as responsible) typically produce significantly longer emotional experiences.

It is plausible to expect an important role for the individual's appraisal processes in the case of bereavement - which is one of the central topics in this special section-due to the abolishment of the bereavement exclusion in the DSM-5 (see Horwitz, 2015). For example the death of a close person is generally an important negative event in one's life, which obstructs the essential need for close relationships, causing many unpleasant consequences. In the face of death, one has no control over the consequences and little coping potential, as even passive adjustment to the new situation is difficult. All this would be accentuated by a sudden death, which often leads to a feeling of injustice of fate. Thus higher intensity and longer duration of the grief episode following such an event would be considered perfectly normal. One would also expect a wide range of duration and intensity across different individuals depending on the severity of the loss and the nature of the appraisal results, especially with respect to perceived coping potential.

It could be argued that shifting the criterion for a normal/ abnormal differentiation from symptoms to appraisal does not advance the field, as it may be just as difficult, if not more, to define the criteria for a disturbed, dysfunctional, unrealistic or inappropriate appraisal process as it is for defining dysfunctional symptoms. This would certainly be the case in trying to apply such a criterion in a one-shot manner to a specific case of appraisal and the consequent emotional reaction. However, it may be possible to identify dispositional biases as potential risk factors for dysfunctional, inappropriate appraisal. To use a wellknown example, extreme pessimists might seriously underestimate their chance of success and extreme optimists might largely overestimate it, leading to failure in both cases. The dispositional bias would lead to dysfunctional decisions and actions, which might well lead to abnormal functioning in the long run. I claim that such appraisal biases might be directly responsible for emotional disturbances.

Here are three empirical examples demonstrating appraisal biases which illustrate this claim:

1. In the Swiss actuarial study referred to before, we asked respondents how often, in general, they experienced several major emotions in their daily life. People who said they frequently experienced sadness, were twice as likely to report that yesterday they experienced a state described by a word from the sadness family. An even stronger effect occurred for anxiety (see Table 4 in Scherer, Wranik, Sangsue, Tran, \& Scherer, 2004). As this was not the case for other emotions it is unlikely to be a simple response tendency effect. Rather it might be a bias to appraise the consequences of events systematically in a biased fashion (e.g., with a helplessness bias), making it more likely to experience sadness or anxiety (although in some cases a long-standing grief situation might be the cause). The identification of such risk factors (using the odds ratio) as demonstrated by Scherer et al. (2004) may be one of the keys to better understand emotion disturbance and malfunctioning.

2. In experimental work in our laboratory (see Wranik \& Scherer, 2010) we used a questionnaire to distinguish between externalizers (who tend to blame others for negative effects) and internalizers (who tend to blame themselves). The experiment consisted of an interaction between two persons trying to jointly solve an unsolvable puzzle. The results showed that the externalizers were significantly more likely to blame the partner for failure to solve the puzzle. While both internalizers and externalizers were equally likely to report anger (independently of the blame), internalizers were more likely to be angry at themselves and externalizers to be angry at their partner. Anger can be of very different kinds and anger towards self and anger towards others could reflect an important appraisal bias that leads to different and dysfunctional emotional responses.

3. We tested 1,457 managers and midlevel employees with a computer assessment instrument (Computer Assessment of Personal Potential [CAPP]; see Scherer, 2007 ) in the context of human resource assessment programs. Individuals scoring $1 S D$ or more above mean on "overexternalization" (mean of scores on external control and external attribution) were significantly: (a) higher on worry/fear in an Emotional Disposition scale, (b) lower on emotional stability in personality tests, and (c) lower on functional coping and higher on repression in a coping inventory (see Scherer, 2007, pp. 109-110). Again this could reflect an appraisal bias that, in extremes, could lead to emotional problems. 
Obviously, appraisal biases are extremely important in any effort to diagnose emotional disturbances. For example, if you have a tendency of underestimating your power to deal with certain events, this may well be a contributing factor to helplessness and depression (Abramson, Seligman, \& Teasdale, 1978). This is a potential appraisal bias with obvious clinical relevance for the depression syndrome but there are many others. Scherer and Brosch (2009) have reviewed this issue and have systematically listed the type of bias and the risk for certain types of emotional disturbance that they might engender. For example a tendency to overestimate the certainty of negative outcomes can lead to pessimism and depressive moods whilst an inability to judge relevant events as important can lead to apathy. We believe that this approach could significantly contribute to advancement on the thorny issue of distinguishing normal and abnormal emotion process (see Mehu \& Scherer, 2015, for a more detailed treatment of appraisal bias, a proposal for empirical measurement, and examples for potential applications).

\section{Conclusions}

In conclusion, I return to the four basic functions of emotion used to illustrate how the normal emotion process can be modeled by the CPM, how such models could inform the understanding of emotional dysfunction, and how the identification of potential risk factors could support clinical judgment (the functional approach is discussed in further detail in the contributions of Gilbert, 2015; Stein \& Nesse, 2015).

1. The CPM model's basic assumption is that emotional processes are driven by multilevel cognitive appraisal to evaluate the event, potential consequences for the individual, and the needs and resources for coping with the situation. The more realistic the appraisal, the more appropriate and adaptive will be the response preparation. Anything that adversely affects that appraisal process such as cognitive deficits, lack of attention, inappropriate associations, memory failure, can lead to dysfunctional appraisal process. Thus, the tendency to ruminate can negatively influence emotion-antecedent appraisal process because of the focus on specific, often inappropriate, elements of a problem (see the contribution by Watkins, 2015). Another important factor relates to appraisal biases as described before such as unrealistic causal attribution or hopes and beliefs that produce tendencies to appraise events in distorted ways. This becomes particularly problematic when schematic appraisal tendencies become automatic and increasingly rigid. Emotion research should focus more on the identification and measurement of such appraisal deficiencies and biases with an attempt to clarify the mechanisms leading from biases to disturbances.

2. Emotions prepare for adaptive action but the behavior itself is not part of the emotion. Rather, it is the result of complex weighing of different inciting and inhibiting factors. Thus, in anger one normally does not immediately hit the person who angered one. Normally, even at an unconscious level, regulation mechanisms kick in when emotionality rises because our system tries to modulate the amount of arousal as this is costly for the organism and considers social rules and potential consequences. However, some people may have deficits in this response modulation mechanism resulting in extreme impulsivity, bipolar disorders, antisocial behavior disorder, or psychopathy. These deficits have of course been intensively studied in psychopathology but it may be of interest to investigate the continuity from control and regulation functions in normal emotions to pathological cases in order to better understand mechanisms and risk factors (including early detection in development).

3. The third function of emotion is component integration, internal monitoring, and conscious representation of the processes in an emotional episode. This is an essential capacity for the regulation of the prospective response to an event as described before because one compares the internal representation with cultural expectations. A deficit in this prospective representation and anticipation is likely to lead to abnormal emotional functioning. It is probably also of central importance to emotional learning and the acquisition of emotion knowledge, a central element of emotional competence. There has been very little research in this domain and there is an urgent need to intensify work on the mechanisms underlying emotion representation.

4. The fourth function concerns emotion communication. Emotions are eminently social and centrally involved in social communication, informing others about our evaluation of certain events and our possible reactions. If emotional communication fails, and the surrounding social group or society starts to notice, this may be a powerful indicator of dysfunctionality. If a person can no longer communicate correctly what he or she is feeling, or to correctly infer what others are feeling and why, social interaction becomes very difficult, as, for example, in the case of autistic spectrum disorders. Emotion researchers have long been concerned with emotion expression, both on the encoding and the decoding side, and many diagnostic instruments exist. Increased contact between emotion researchers and clinicians might help to exploit these resources to identify and diagnose lack of competence in this domain and possibly develop remedial measures.

Given the underlying mechanisms briefly enumerated here, it is obvious that emotion processes are extremely variable even within the normal domain. There is a large degree of individual difference with people reacting differently to the same situation, due to major differences in event appraisal and response mechanisms, resulting in major variations in terms of duration, intensity or quality of distinct experiences. The normal gamut of emotional responding is vast and the borders between them are fuzzy. This is a central problem in distinguishing between 
normal and abnormal emotion and diagnosing affective disorders. It accounts for the problems in trying to classify affective disorders on the basis of typical symptom lists, as nicely illustrated by the transdiagnostic approach (see contribution by Watkins, 2015) and other proposals (e.g., Borsboom \& Cramer, 2013).

Currently, psychology has no ready-made solution to offer. I have argued in this piece that there is a need to better understand normal emotion processes and apply our insights to the issue of diagnosing dysfunctions and their origin. As suggested before, the diagnosis of an emotional disturbance should be directly related to the degree of justification of the emotional investment, as determined by, among other factors, the nature of the individual's life circumstances, the nature of the events he or she has been experiencing, and the appropriateness of the appraisal processes that underlie the emotional responses.

We need more research that adopts a functional point of view, identifying cases which do not facilitate adaptation to an ever changing social world. Admittedly, this is a difficult issue as it can be shown that seemingly pathological emotional behavior can be functional for the respective individual (overresponsive reactions maybe highly adaptive because they are more "inexpensive" given the stakes; Nesse, 2001). However, such examples should not lead us to reject functional approaches. Rather, it might be a good strategy to choose mechanisms that lend themselves to discover malfunctioning, like appraisal biases that block a realistic assessment of the consequences of an event and our coping potential, preventing the emotion mechanism to serve its adaptation function. As such it may be useful to consider emotions to be dysfunctional when the investment does not justify the expenditure, in terms of depleting both physical and psychological resources, as this has the potential to exhaust and eventually harm the individual in the long term.

As to the efficient diagnosis of emotional dysfunctions, the very existence of a lively debate, further fueled by the decisions made by DSM-5, is a promising development. Emotion researchers could contribute to progress in this area by more extensive investigation of underlying emotion mechanisms and potential dysfunctions leading to reduced emotional competence. Given such advances in our knowledge about normal emotion processes, clinicians begin to find it useful to integrate such knowledge into their diagnostic expertise.

\section{Declaration of Conflicting Interests}

None declared.

\section{Note}

1 The Swiss Household Panel (SHP) is based at the Swiss Centre of Expertise in the Social Sciences FORS. The project is financed by the Swiss National Science Foundation.

\section{Supplementary Material}

The online data supplements are available at http://emr.sagepub. com/supplemental.

\section{References}

Abramson, L. Y., Seligman, M. E. P., \& Teasdale, J. D. (1978). Learned helplessness in humans: Critique and reformulation. Journal of Abnormal Psychology, 87(1), 49-74.

Bondolfi, G., Mazzola, V., \& Arciero, G. (2015). In between ordinary sadness and clinical depression. Emotion Review, 7, 216-222.

Borsboom, D., \& Cramer, A. O. J. (2013). Network analysis: An integrative approach to the structure of psychopathology. Annual Review of Clinical Psychology, 9, 91-121.

Brosch, T., Sander, D., \& Scherer, K. R. (2007). That baby caught my eye... Attention capture by infant faces. Emotion, 7(3), 685-689.

Darwin, C. (1998). The expression of emotions in man and animals (3rd ed., P. Ekman, Ed.). London, UK: HarperCollins. (Original work published 1872)

Ellsworth, P. C., \& Scherer, K. R. (2003). Appraisal processes in emotion. In R. J. Davidson, K. R. Scherer, \& H. Goldsmith (Eds.). Handbook of the affective sciences (pp. 572-595). New York, NY: Oxford University Press.

Fontaine, J. R. J., Scherer, K. R., \& Soriano, C. (Eds.). (2013). Components of emotional meaning: A sourcebook. Oxford, UK: Oxford University Press.

Frijda, N., \& Scherer, K. R. (2009). Emotion definitions (psychological perspectives). In D. Sander \& K. R. Scherer (Eds.), Oxford companion to emotion and the affective sciences (pp. 142-144). Oxford, UK: Oxford University Press.

Gilbert, P. (2015). An evolutionary approach to emotion in mental health with a focus on affiliative emotions. Emotion Review, 7, 230-237.

Goffman, E. (1963). Stigma: Notes on the management of spoiled identity. Englewood Cliffs, NJ: Prentice-Hall.

Horwitz, A. V. (2015). The DSM-5 and the continuing transformation of normal sadness into depressive disorder. Emotion Review, 7, 209-215.

Leventhal, H., \& Scherer, K. R. (1987). The relationship of emotion to cognition: A functional approach to a semantic controversy. Cognition and Emotion, 1, 3-28.

Mehu, M., \& Scherer, K. R. (2015). The appraisal bias model of cognitive vulnerability to depression. Emotion Review, 7, 272-279.

Nesse, R. M. (2001). The smoke detector principle: Natural selection and the regulation of defenses. Annals of the New York Academy of Sciences, 935, 75-85.

Öhman, A., \& Mineka, S. (2001). Fears, phobias, and preparedness: Toward an evolved module of fear and fear learning. Psychological Review, 108, 483-522.

Rimé, B. (2009). Emotion elicits the social sharing of emotion: Theory and empirical review. Emotion Review, 1, 60-85.

Scherer, K. R. (2001). Appraisal considered as a process of multi-level sequential checking. In K. R. Scherer, A. Schorr, \& T. Johnstone (Eds.), Appraisal processes in emotion: Theory, methods, research (pp. 92-120). New York, NY: Oxford University Press.

Scherer, K. R. (2004). Feelings integrate the central representation of appraisal-driven response organization in emotion. In A. S. R. Manstead, N. H. Frijda, \& A. H. Fischer (Eds.), Feelings and emotions: The Amsterdam Symposium (pp. 136-157). Cambridge, UK: Cambridge University Press.

Scherer, K. R. (2005a). Unconscious processes in emotion: The bulk of the iceberg. In P. Niedenthal, L. Feldman-Barrett, \& P. Winkielman (Eds.), The unconscious in emotion (pp. 312-334). New York, NY: Guilford.

Scherer, K. R. (2005b). What are emotions? And how can they be measured? Social Science Information, 44(4), 693-727.

Scherer, K. R. (2007). Component models of emotion can inform the quest for emotional competence. In G. Matthews, M. Zeidner, \& R. D. Roberts (Eds.), The science of emotional intelligence: Knowns and unknowns (pp. 101-126). New York, NY: Oxford University Press. 
Scherer, K. R. (2009). The dynamic architecture of emotion: Evidence for the component process model. Cognition and Emotion, 23(7),1307-1351.

Scherer, K. R., \& Brosch, T. (2009). Culture-specific appraisal biases contribute to emotion dispositions. European Journal of Personality, 23, 265-288.

Scherer, K. R., \& Meuleman, B. (2013). Human emotion experiences can be predicted on theoretical grounds: Evidence from verbal labeling. PLoS ONE, 8(3), e58166. doi:10.1371/journal.pone.0058166

Scherer, K. R., \& Wallbott, H. G. (1994). Evidence for universality and cultural variation of differential emotion response patterning. Journal of Personality and Social Psychology, 66, 310-328.

Scherer, K. R., Wallbott, H. G., \& Summerfield, A. B. (Eds.). (1986). Experiencing emotion: A cross- cultural study. Cambridge, UK: Cambridge University Press.

Scherer, K. R., Wranik, T., Sangsue, J., Tran, V., \& Scherer, U. (2004). Emotions in everyday life: Probability of occurrence, risk factors, appraisal and reaction pattern. Social Science Information, 43(4), 499-570.

Stein, D. J., \& Neese, R. M. (2015). Normal and abnormal anxiety in the age of DSM-5 and ICD-11. Emotion Review, 7, 223-229.

Stroebe, M., Schut, H., \& Stroebe, W. (2007). Health outcomes of bereavement. Lancet, 370, 1960-1973.
Van Reekum, C. M., \& Scherer, K. R. (1997). Levels of processing for emotion-antecedent appraisal. In G. Matthews (Ed.), Cognitive science perspectives on personality and emotion (pp. 259-300). Amsterdam, The Netherlands: Elsevier Science.

Verduyn, P., van Mechelen, I., Tuerlinckx, F., \& Scherer, K. R. (2013). The relation between appraised mismatch and the duration of negative emotions: Evidence for universality. European Journal of Personality. Advance online publication. doi:10.1002/per.1897

Wallbott, H. G., \& Scherer, K. R. (1986). The antecedents of emotional experience. In K. R. Scherer, H. G. Wallbott, \& A. B. Summerfield (Eds.), Experiencing emotion: A cross- cultural study (pp. 69-83). Cambridge, UK: Cambridge University Press.

Watkins, E. (2015). An alternative transdiagnostic mechanistic approach to affective disorders illustrated with research from clinical psychology. Emotion Review, 7, 250-255.

Wranik, T., \& Scherer, K. R. (2010). Why do I get angry? A componential appraisal approach. In M. Potegal, G. Stemmler, \& C. Spielberger, (Eds.). International handbook of anger: Constituent and concomitant biological, psychological, and social processes (pp. 243-266). New York, NY: Springer. 